\title{
Immunohistologic Analysis of a Human Pulmonary Alveolar Macrophage Antigen ${ }^{1}$
}

\author{
Lester Kobzik, John J. Godleski, Andrea Biondi, ${ }^{*} 2$ Carl J. O'Hara, $\dagger$ \\ AND ROBERT F. TODD III*,‡ \\ Departments of Pathology, Brigham \& Women's and $\dagger$ New England Deaconness Hospitals, the \\ *Division of Tumor Immunology, Dana-Farber Cancer Institute, Boston, Massachusetts 02115, and \\ $\ddagger$ the Department of Internal Medicine, University of Michigan Medical School, \\ Ann Arbor, Michigan 48109
}

\begin{abstract}
PAM1 is a 200-kDa polypeptide antigen present on lavaged human alveolar macrophages but not on monocytes, peritoneal macrophages, breast milk macrophages, or other normal hematopoietic cells studied by flow cytometry. We have characterized the distribution of expression of this antigen by cells in tissues by using immunohistologic techniques. Normal and diseased lung as well as lymph nodes, spleen, kidney, liver, GI tract, and skin were studied. PAMl was expressed strongly on the surface and weakly in the cytoplasm of most alveolar macrophages in all 15 of the lung specimens. Occasional interstitial macrophages had weak to moderate staining for this antigen but the majority did not stain. The distribution, pattern, and intensity of staining for PAM1 was the same in normal lung specimens and those with interstitial pneumonitis, despite the increase in mononuclear cells in the latter. Dermal histiocytes and Kuppfer cells expressed PAM1 weakly. Sinus histiocytes in lymph nodes were moderately to strongly positive. Although lymphoid cell suspensions (tonsil) were negative by flow cytometry, five of six lymph nodes had positive cells by immunohistology. PAM1 was also detected on endothelial cells of splenic sinusoids in all 6 specimens but not on any other endothelium. Hence, while PAM1 is expressed most strongly on alveolar macrophages, it can also be demonstrated in other locations using sensitive immunohistologic techniques. Since circulating monocytes are antigen negative and some lung interstitial macrophages bear antigen, PAM1 may be a useful marker for studies of the differentiation of mononuclear cells in the lung. 1985 Academic Press. Inc.
\end{abstract}

\section{INTRODUCTION}

Cell surface antigens detected with specific monoclonal antibodies have the potential to be important markers of differentiation and heterogeneity within the mononuclear phagocyte system (1). Antigenic determinants have been described which are unique to mononuclear phagocytes in specific anatomic locations (1-4). Among these, the surface antigens of alveolar macrophages are of particular interest because of the role these cells play in the pathogenesis of lung diseases (5).

A human pulmonary alveolar macrophage antigen, designated PAM1, was recently described using a mouse monoclonal antibody generated by immunization with human alveolar cells (4). This antigen was shown to be a $200-\mathrm{kDa}$ polypeptide

\footnotetext{
1 This work was supported by Grants HL27244, T32HL07066, CA39064, and Project Oncology, CNR, Italy.

${ }^{2}$ Present address: Instituto di Ricerche Farmacologiche Mario Negri, Milan, Italy.
} 
present on alveolar macrophages in lavage specimens from both normal and diseased subjects but was not detected on monocytes, peritoneal macrophages, breast milk macrophages, nor on a variety of other normal human hematopoietic cells studied as cell suspensions by flow cytometry.

The purpose of this study was to characterize the tissue distribution of this pulmonary alveolar macrophage antigen and also to examine the relationship of antigen-positive (or negative) cells to normal or pathologic structures. We used immunohistochemical techniques on cryostat sections of a variety of human tissues both to establish whether this antigen is found uniquely in the lung as well as to determine the distribution of cells with this antigen in interstitial pulmonary disease.

\section{METHODS}

Tissue specimens. Samples of human biopsy specimens were collected at the operating room as soon as the tissue was available to the pathologist. The tissues were embedded in OCT compound (Ames Co., Elkhart, Ind.), snap-frozen in isopentane and dry ice, and stored under isopentane at $-70^{\circ} \mathrm{C}$. Six of the lung specimens were taken from uninvolved areas in resections for benign or malignant neoplasms; these were unremarkable histologically and were considered to represent normal lung tissue. Nine other lung specimens were from biopsy specimens removed for inflammatory lung diseases; histologically, these cases all shared the diagnosis of interstitial pneumonitis. Other tissues studied included: lymph nodes (6), spleen (6), kidney (2), liver (2), GI tract (3), bronchus (1), skin (1), and thymoma (1). The lymph nodes were from cervical (3), axillary (2), or inguinal (1) regions and were histologically normal or reactive. The kidney, liver, GI tract, skin, and four of six spleen specimens were also normal. One spleen showed congestive fibrosis; another was infiltrated by lymphoma.

Antibodies. The IgG1 mouse monoclonal anti-PAM1 was used at $10 \mu \mathrm{g} / \mathrm{ml}$ after protein A affinity purification (6). The primary control reagent used was an IgG1 mouse myeloma protein in ascites (BRL, Gaithesburg, Md.) diluted to the same concentration of immunoglobulin as the anti-PAM1. Peroxidase-conjugated rabbit anti-mouse immunoglobulin and peroxidase-conjugated swine anti-rabbit (both, Accurate Chemical Co., N.Y.) were diluted to $1 / 30$ and $1 / 50$, respectively, in the same aliquot. All dilutions were made in phosphate-buffered saline (PBS), $\mathrm{pH} 7.6$.

Immunoperoxidase protocol. Cryostat sections $(8 \mu \mathrm{m})$ of the respective tissues were cut and placed in cold $4 \%$ buffered paraformaldehyde (7). After fixation for 10-15 min, the sections were washed with PBS and then stained using the immunoperoxidase protocol of Kurtin and Pincus (8). Sections were sequentially incubated with the following: (1) $1 \%$ rabbit serum in PBS to block nonspecific binding; (2) primary monoclonal antibody or control; (3) $1.5 \%$ hydrogen peroxide in methanol to block endogenous peroxidase; (4) a secondary antibody complex of peroxidase-conjugated rabbit anti-mouse and swine anti-rabbit prepared 30-60 min prior to use. All incubations lasted for $30 \mathrm{~min}$ and were followed by repeated washes in PBS. Final staining was achieved by incubation with a peroxidase substrate solution of $.01 \%$ hydrogen peroxide and $0.05 \%$ diaminobenzidine in 0.05 
M Tris buffer, $\mathrm{pH} 7.3$, followed by counterstaining with hematoxylin or methyl green. Studies on each tissue sample were replicated at least three times.

\section{RESULTS}

In order to analyze the distribution of PAM1 antigen expression by cells within various human tissues, we used a modified immunoperoxidase technique (8). This produced clear, easily discernible staining, was relatively quick and simple, and showed minimal background staining. Control sections (stained with negative control IgG1 antibody) of lung and all other tissues showed no staining whatsoever. An important modification was the use of a higher concentration (1.5 vs $0.3 \%$ ) of hydrogen peroxide in methanol to block the high amounts of endogenous peroxidase-like activity in lung macrophages and in spleen. This step was used after primary antibody incubation without diminution of antigenicity of the positive cells.

Using this immunoperoxidase staining protocol, we examined the following human tissues for expression of the PAM1 marker.

Lung. In the 6 normal lung specimens PAM1 was present on almost all the alveolar macrophages. Although cytoplasmic staining was noted, cell surface staining was clearly more prominent (Fig. 1). Control sections were negative (Fig. 2). Some heterogeneity in the intensity of staining was seen within clusters of macrophages in a given alveolus or area of tissue, but the variation was usually from moderate to strong staining. Weak to moderate staining for PAM1 was also seen in a small proportion of interstitial cells that had the morphologic appearance of macrophages (Fig. 3); most such cells were antigen negative. Examination of the 9 lung specimens with interstitial pneumonitis showed no difference in the distribution or intensity of positive staining for PAM1, despite the greater number of alveolar macrophages in these specimens (Fig. 4). No staining of any other cell type was noted in the 15 normal or diseased lung specimens nor in one specimen of bronchus.

Lymph nodes. In five of six lymph nodes, sinus histiocytes in both subcapsular and medullary areas were positive for PAM1 (Fig. 5). Other cells in the cortex and medulla were negative for antigen. The proportion of histiocytes positive for antigen varied from approximately 5 to $50 \%$. Positive staining was seen in an inguinal lymph node as well as in cervical and axillary nodes. One lymph node showed no PAM1 positive cells whatsoever on repeated studies.

Liver. In both liver specimens slender Kupffer cells lining hepatic sinusoids showed focal weak staining. All other cell types were negative.

Skin. Dermal histiocytes were weakly positive in the normal skin specimen studied. No other epidermal or dermal cells showed positivity.

Spleen. In six of six spleen specimens PAM1 antigen was detected on the endothelial cells of the red pulp sinusoids (Fig. 6). All of the endothelial cells of these sinusoids appeared equally stained in each specimen, with variations from weak to moderate staining from specimen to specimen. Both cytoplasmic and membrane staining were seen, as in other sites. Other vessels in the white pulp or trabeculae, as well as all other cells of the spleen, were negative for antigen.

Other tissues. Kidney (2), GI tract (3), including appendix with prominent 

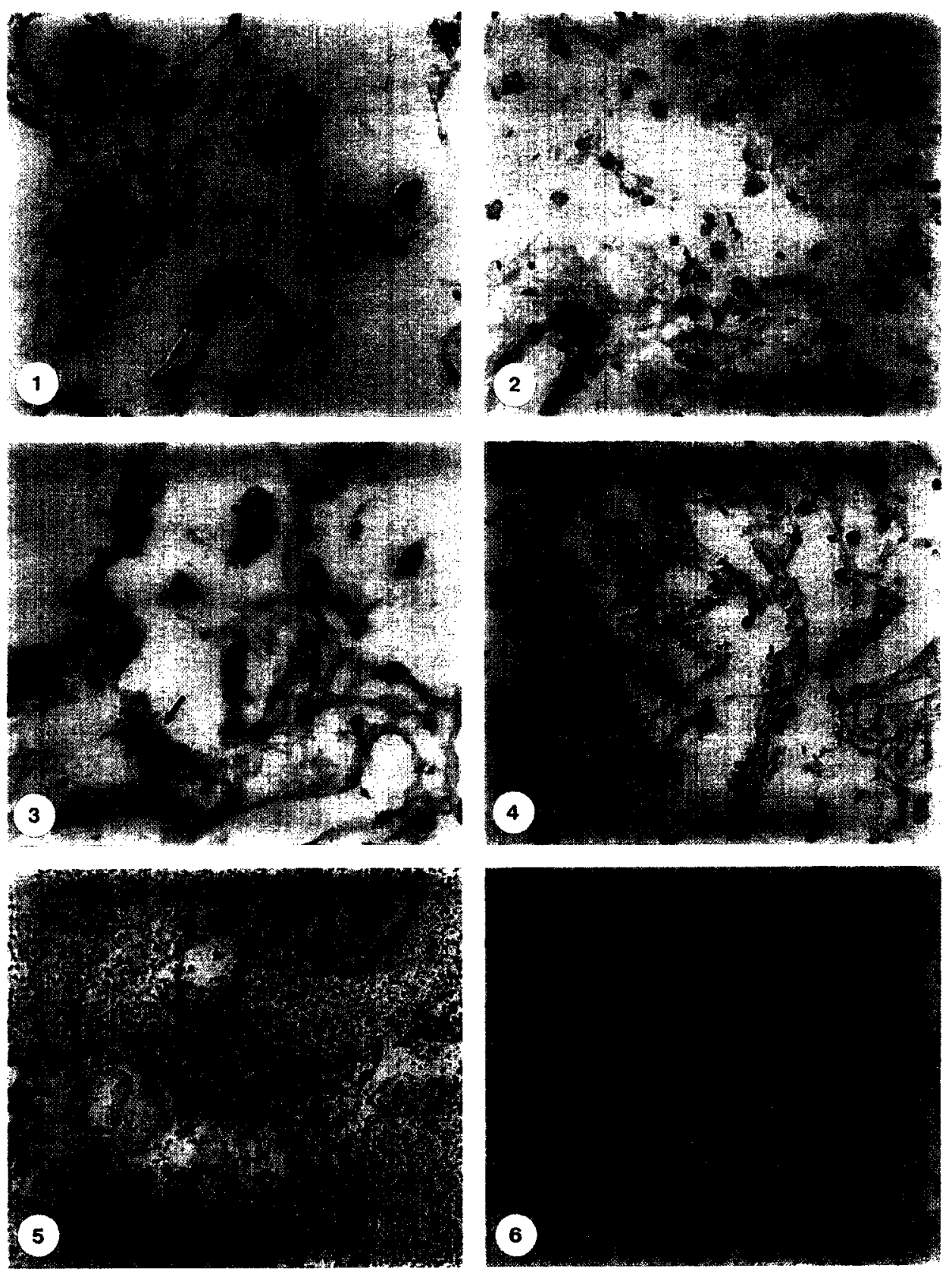

FIG. 1. Alveolar macrophages show strong cell surface and some cytoplasmic staining for PAM I. Other cell types show no staining $(\times 280)$.

FIG. 2. Control sections stained with nonspecific antibody showed no positive staining $(\times 175)$.

FIG. 3. Occasional interstitial macrophages were positive for PAM1 (arrows), but most were negative $(\times 280)$.

FIG. 4. Areas of lung tissue with interstitial pneumonitis show an increased number of macrophages, but the distribution and intensity of antigen positive cells is the same as in normal lung $(\times 175)$.

FIG. 5. PAM1 positive histiocytes were found in subcapsular and medullary sinuses of lymph nodes $(\times 175)$.

FIG. 6. The endothelium of red pulp sinusoids showed weak to moderate staining for PAM1. All other spleen cells were negative $(\times 175)$. 
TABLE 1

\begin{tabular}{llcl}
\hline \multicolumn{1}{c}{ Organ } & \multicolumn{1}{c}{ Cell type positive } & Proportion positive $^{a}$ & \multicolumn{1}{c}{ Intensity $^{c}$} \\
\hline Lung (15) & Alveolar macrophage & $>90 \%$ & Strung \\
& Interstitial macrophage & $<5 \%$ & Weak \\
Lymph nodes (6) & Sinus/medullary histiocyte & $5-50 \%$ & Moderate \\
Liver (2) & Kupffer cell & $-10 \%$ focal & Weak \\
Skin (1) & Dermal histiocytes & $\simeq 25 \%$ & Weak \\
Spleen (6) & Endothelium, sinusoidal & $90 \%$ & Weak-moderate \\
Kidney (2) & None & - & - \\
GI tract (3) & None & - & - \\
Thymoma (1) & None & - & - \\
\hline
\end{tabular}

a Estimates after survey of triplicate slides.

${ }^{b}$ Number of specimens examined.

c Endothelium in all other tissues was negative.

lymphoid follicles and thymoma (1), were all negative for PAM1. Table 1 summarizes the data for the distribution of PAM1.

\section{DISCUSSION}

Immunohistologic studies of the tissue distribution of cells bearing specific antigens are important correlates to studies of cell suspensions by immunofluorescence using flow cytometry or microscopy. These studies frequently have incongruous results because of numerous differences between the two techniques $(9,10)$. Moreover, in situ immunologic staining allows direct correlation between antigen expression and cellular morphology and is sensitive enough for the identification of small subpopulations of antigen-positive cells. The sensitivity of immunoenzymatic detection of antigens is enhanced due to the greater amplification by secondary or tertiary antibodies when compared to usual immunofluorescence protocols, and thus small subpopulations of positive cells can be more easily detected. Conversely, preparation of tissues for flow cytometric analysis may give rise to selective cell losses with the possible exclusion of antigen-positive cells. Finally, the presence or absence of antigens in the cytoplasm of cells is best assessed in tissue sections.

We have determined the tissue distribution of cells bearing PAM1, an antigen found only on human alveolar macrophages in flow cytometry studies. While we found most alveolar macrophages to be strongly positive for the antigen, we also noted its presence on other cells. In the lung, infrequent interstitial cells positive for PAM1 were seen in both normal and diseased specimens. Other cell types within the lung, including epithelium and endothelium, were uniformly negative. Areas of interstitium that were expanded as a result of acute or chronic inflammation did not show an appreciable increase in PAM1 positive cells although more cells with the morphologic appearance of macrophages were present. It is unlikely that technical factors such as fixative or antibody penetration to antigen within tissue can account for the lesser staining of interstitial lung macrophages, since distinct PAM1 positivity was detectable in other tissue sites processed in the same way.

Outside the lung we found PAM1 present on sinus histiocytes of five of six 
lymph nodes examined. This included an inguinal lymph node, arguing against presence of PAM1 only in nodes draining upper or lower respiratory tract. Flow cytometry on lymphoid cell suspensions from tonsil or spleen for PAM1 was essentially negative (4). Kupffer cells and dermal histiocytes were also weakly positive with our technique. Our finding of PAM1-positive cells in these tissues provides another example of the utility of immunohistologic studies in supplementing cell suspension data.

Immunohistochemical techniques have shown that some mononuclear phagocyte antigens are also present on endothelial cells $(2,12)$. Indeed PAM1 was detected on endothelium in six of six spleen tissue samples tested. Weak to strong staining was present on the endothelial lining of red pulp sinusoids, with no staining of other endothelial components in the spleen or in any other tissue. Antigens shared by cells of the mononuclear phagocyte system and endothelium have been previously reported (13). Recently, the antibody OKM5 was shown to detect an antigen present on circulating monocytes and a variety of endothelial beds (12). Similarly, PHM2 which stains monocytes, some T cells, and macrophages in many tissue sites, was reported by Hancock et al. to stain endothelium in lymph node, spleen, thymus, and kidney (2). Some heterogeneity in the expression of OKM5 by different vascular beds was noted by Knowles et al. (12). The restricted expression of PAM1 to the sinusoids of an important lymphoid organ, the spleen, is especially intriguing in the context of recent information suggesting that endothelial cells function in immune processes (14). Further clarification of the significance of this shared expression of PAM1 awaits determination of the function of this antigen.

Although PAM1 is found on cells other than alveolar macrophages, it still appears to have its most concentrated expression on lung macrophages which are more terminally differentiated. Since monocytes are antigen negative and some antigen can be detected within lung interstitium, PAM1 may be a useful marker for studies of the involvement of the interstitium in alveolar macrophage maturation in man. The role of monocytes and lung interstitial cells in the generation of alveolar macrophages has long been an area of active investigation (15). Studies in animals using tritiated thymidinc labeling and autoradiography indicate that the majority of alveolar macrophages are derived from blood monocytes (16). Some, but not all, investigators find evidence for a small contribution from precursors dividing in the lung interstitium (17). Immunologic studies of macrophage antigens present on lung macrophages but not on circulating monocytes provide another approach to these issues. For example, in studies of a hamster lung macrophage-specific antigen, amount of antigen expression was correlated with the age of lung macrophage subpopulations (18). Decreased antigen as assayed by flow cytometry correlated with recent DNA synthesis. In contrast, alveolar macrophages expressing large amounts of this antigen had synthesized DNA at least 5 days before assay. This indicates an antigenic differentiation parallel to other functional and metabolic changes in these cells (5). Decreased expression of hamster lung macrophage specific antigen has also been noted in alveolar macrophages from animals with acute or chronic lung inflammation reflecting a significant proportion of immature macrophages recruited into these diseased lungs (19). 
If PAM1 antigen increases after cells have passed through the interstitium, immunohistologic studies of lung tissue, including interstitium, can provide another way to examine mononuclear phagocytes in the transition phase from circulation to the alveolus. Previous studies of macrophage antigen expression in tissue sections have not reported results in the interstitium, even when alveolar macrophages are positive for the antigen under study $(2,5,12)$. Our data indicate that at least some human lung interstitial macrophages express PAM1 weakly. Based on the kinetic studies cited above, we suggest that these cells are in transition from monocyte to alveolar macrophage. Although few studies suggest that alveolar macrophages migrate back into interstitium, we cannot completely exclude the possibility that these cells are in transit from the alveolar surface to pulmonary lymphoid tissue (20). Nevertheless, we speculate that these findings reflect the differentiation program of monocytes destined to become alveolar macrophages and may indicate a role for some component(s) of the lung interstitium in regulating or initiating such antigenic and functional differentiation. In vitro studies of PAM1 modulation by isolated elements of the lung interstitium will allow testing of these hypotheses.

\section{REFERENCES}

1. Todd, R. F, and Schlossman, S. F., In "Immunology of the Reticuloendothelial System: A Comprehensive Treatise"' (Bellanti, J. A., Herscowitz, H. B., Eds.), Vol. 6, pp. 87-112. Plenum, New York, 1984.

2. Hancock, W. W., Zola, H., and Atkins, R. C., Blood 62, 1271, 1983.

3. Rumpold, H., Forsten, O., Bock, G., Swetley, P., and Reidl, M., Immunology 45, 637, 1982.

4. Biondi, A., Rossing, T. H., Bennett, J., and Todd, R. F., J. Immunol. 132, 1237, 1984.

5. Hocking, W. G., and Golde, D. W., N. Engl. J. Med. 301, 580, 1979.

6. Bywater, R, , In "Chromatography of Synthetic and Biological Polymers" edited by (Epton, R., Ed.), pp. 337-340. Ellis Horwood, Chichester, U.K., 1978.

7. Hancock, W. W., Becker, G. J., and Atkins, R. C., Amer. J. Clin. Pathol. 78, 825, 1982.

8. Kurtin, P. J., and Pincus, G. S., Human Pathol. 16, 353, 1985.

9. Flotte, T. J., Springen, T. A., and Thorbecke, G. J., Amer. J. Pathol. 111, 112, 1983.

10. Borowitz, M. F., Croker, B. P., and Metzger, R. S., Amer. J. Pathol. 105, 97, 1981.

11. Mason, D. Y., and Biberfeld, P., J. Histochem. Cytochem. 28, 731, 1980.

12. Knowles, D. M., Tolidjian, B., Marboe, C., D'Agati, V., Grimes, M., and Chess, L., J. Immunol. 132, $2170,1984$.

13. Stastny, P., Transplant. Proc. 12(Suppl. 1), 32, 1980.

14. Pober, J. S., Gimbrone, M. A., Cotran, R. S., Reiss, C. S., Burakoff, S. J., Fiers, W., and Ault, K. A., J. Exp. Med. 157, 1339, 1983.

15. Brain, J. D., Godleski, J. J., and Sorokin, S. P., In "Respiratory Defense Mechanisms" (Brain, J. D., Proctor, D. F., Reid, L. M., Eds.), pp. 849-892. Marcel Dekker, New York, 1977.

16. Alblas, A. B. V., and Van Furth, R., J. Exp. Med. 149, 1504, 1979.

17. Bowden, D. H., and Adamson, I. Y. R., Lab. Invest. 42, 511, 1980.

18. Harbison, M. L., Godleski, J. J., Mortara, M., and Brain, J. D., Lab. Invest. 50, 653, 1984.

19. Tryka, A. F., Godleski, J. J., Katler, M., and Brain J. D., Exp. Lung Res., 7, 41, 1984.

20. Corry, D., Kulkarni, P., and Lipscomb, M. F., Amer. J. Pathol. 115, 321, 1984.

Received March 25, 1985; accepted with revision May 22, 1985 